\title{
Câncer de Mama e Eritrodermia: Relato de Caso
}

doi: https://doi.org/10.32635/2176-9745.RBC.2021v67n1.1156

\section{Breast Cancer and Erythroderma: Case Report \\ Cáncer de Mama y Eritrodermia: Relato de Caso}

Maykom de Lira Barbosa'; Yara Ayami Mattos Abe²; Fábio de Queiroz Medeiros³; Hilka Flávia Barra do Espírito Santo Alves Pereira4

RESUMO

Introduçáo: A eritrodermia esfoliativa é um evento raro que está associado a diversas doenças como psoríase, eczemas, neoplasias malignas, uso de medicamentos, entre outras. Relato do caso: Paciente de 63 anos apresentou quadro de exantema difuso iniciado em janeiro de 2018, evoluindo com descamação generalizada da pele e formação de úlceras, sentindo calafrios. Não referiu histórico de câncer na família. $\mathrm{Na}$ anamnese, não houve relato de nenhum tipo de eczema, doença de pele preexistente ou uso de plantas medicinais. Foi identificada neoplasia maligna de mama do subtipo molecular luminal A, posteriormente. Após mastectomia com linfadenectomia, houve apenas melhora parcial do quadro da eritrodermia. Atualmente, em uso de doxorrubicina. Conclusáo: A paciente apresentou quadro clínico inicial condizente com o que se espera de eritrodermia, que pode estar associada ao surgimento de neoplasia maligna de mama. O presente relato é importante, pois pode auxiliar em diagnósticos diferenciais para a eritrodermia, mesmo na vigência de um quadro clínico atípico. Palavras-chave: Neoplasias da Mama; Dermatite Esfoliativa; Mastectomia Radical.

\section{ABSTRACT}

Introduction: Exfoliative erythroderma is a rare event associated with several diseases such as psoriasis, eczema, malignant neoplasms, medication use, among others. Case report: A 63-year-old patient presented diffuse rash that started in January 2018, evolving with generalized skin desquamation and ulcer formation, with chills. Did not report family history of cancer. In the anamnesis, there was no report of any type of eczema, preexisting skin disease or use of medicinal plants. Malignant breast cancer of luminal A molecular subtype was identified later. After mastectomy with lymphadenectomy, there was only partial improvement in erythroderma. Currently using doxorubicin. Conclusion: The patient presented an initial clinical condition consistent with what is expected from erythroderma, which may be associated with the emergence of malignant breast neoplasm. The present report is important because it can help in differential diagnoses for erythroderma, even in the presence of an atypical clinical case.

Key words: Breast Neoplasms; Dermatitis, Exfoliative; Mastectomy, Radical.

\section{RESUMEN}

Introducción: La eritrodermia exfoliativa es un evento raro asociado con varias enfermedades como psoriasis, eccema, neoplasmas malignos, uso de medicamentos, entre otros. Relato del caso: Paciente de 63 ańos presentó una erupción cutánea difusa que comenzó en enero de 2018 , que evolucionó con descamación generalizada de la piel y formación de úlceras, con escalofríos. No informó antecedentes familiares de cáncer. En la anamnesis, no hubo informes de ningún tipos de eccema, enfermedad cutánea preexistente o uso de plantas medicinales. La neoplasia de mama maligna del subtipo molecular luminal A se identificó más tarde. Después de la mastectomía con linfadenectomía, solo hubo una mejoría parcial en la eritrodermia. Actualmente usa doxorrubicina. Conclusión: La paciente presentó un cuadro clínico inicial consistente con lo que se espera de la eritrodermia, que puede estar asociada con la aparición de neoplasma maligno de mama. El presente informe es importante porque puede ayudar en los diagnósticos diferenciales de eritrodermia, incluso en presencia de un cuadro clínico atípico.

Palabras clave: Neoplasias de la Mama; Dermatitis Exfoliativa; Mastectomía Radical.

1,2Universidade Federal do Amazonas (UFAM). Manaus (AM), Brasil.

${ }^{3,4}$ Fundação Centro de Controle de Oncologia do Amazonas (FCecon). Manaus (AM), Brasil.

'Faculdade de Medicina. E-mail: maykomlira@gmail.com. Orcid iD: https://orcid.org/0000-0003-3002-2326

${ }^{2}$ Faculdade de Fisioterapia. E-mail: yara.abe02@gmail.com. Orcid iD: https://orcid.org/0000-0002-6208-6845

${ }^{3}$ Departamento de Oncologia Clínica. E-mail: fqmedeiros@yahoo.com.br. Orcid iD: https://orcid.org/0000-0002-8560-9987

${ }^{4}$ Departamento de Mastologia. E-mail: hilkaespiritosanto@me.com. Orcid iD: https://orcid.org/0000-0002-8028-3199

Endereço para correspondência: Maykom de Lira Barbosa. Rua Aluísio Azevedo, 1137 - Japiim. Manaus (AM), Brasil. CEP 69078-562. E-mail: maykomlira@gmail.com 


\section{INTRODUÇÃO}

A eritrodermia (dermatite esfoliativa) consiste em um quadro sindrômico que se caracteriza por erupção universal eritematosa e descamativa, é um padrão de manifestação cutânea de inúmeras doenças, incluindo psoríase, eczema, neoplasias malignas ou uso de medicamentos. Aproximadamente $80 \%$ a $90 \%$ da superfície cutânea é acometida, com certo grau de edema e prurido quase sempre presente ${ }^{1}$. É considerada uma doença rara, com incidência de 1 a 70 por 100 mil pacientes dermatológicos. Diante de um quadro de eritrodermia, é de suma importância que o médico descarte neoplasia como causa, pois pode se tratar de uma síndrome paraneoplásica, apesar de estudos apontarem que pode corresponder a menos de $1 \%$ dos $\operatorname{casos}^{2,3}$.

Apesar dos baixos índices de mortalidade, a morbidade relacionada com a dermatite esfoliativa é considerável, por ser muitas vezes de evolução crônica, com sinais e sintomas debilitantes, como prurido e descamação intensos, implicando na relevância do diagnóstico precoce, possibilitando intervenção de maneira apropriada e antecipada, de acordo com a etiopatogenia de cada caso ${ }^{4}$.

Este estudo possui como objetivo relatar o caso de uma paciente portadora de neoplasia maligna de mama com um quadro de eritrodermia. A revisão bibliográfica foi realizada para obter uma discussão mais abrangente e detalhada sobre o tema.

\section{MÉTODO}

O trabalho consiste em um estudo de caso de indivíduo único, no qual a seleção da paciente se deu de acordo com os achados clínicos, radiológicos e histopatológicos encontrados por exame físico e no prontuário, sendo realizado na Fundação Centro de Controle de Oncologia do Amazonas (FCecon). A pesquisa iniciou-se mediante aprovação pelo Comitê de Ética em Pesquisa da Universidade Federal do Amazonas (CAAE: 16066619.0.0000.5020) e assinatura do Termo de Consentimento Livre e Esclarecido (TCLE).

\section{RELATO DO CASO}

Paciente do sexo feminino, 63 anos e 10 meses, aposentada, viúva, procedente de Manaus-AM, ensino fundamental incompleto e parda. Em janeiro de 2018, iniciou um quadro súbito de prurido em extremidades com escoriações e descamação; após alguns dias, evoluiu para quadro de exantema difuso e onicólise, fazendo com que procurasse serviço de dermatologia de uma unidade de referência, onde iniciou uso de anti-histamínicos (loratadina) e pomada à base corticosteroides para diminuir o prurido em razão da suspeita de reação alérgica, entretanto, não houve melhora. A paciente negou doenças alérgicas concomitantes ou alergia a medicamentos, negou diabetes mellitus, porém apresentava diagnóstico de hipertensão arterial sistêmica. Não referiu histórico de câncer na família. $\mathrm{Na}$ anamnese, não houve relato de nenhum tipo de eczema, doença de pele preexistente ou uso de plantas medicinais, incluindo qualquer medicamento tópico utilizado anteriormente.

Em maio de 2018, realizou mamografia e recebeu o resultado com a presença de nódulos sólidos irregulares na mama direita e linfonodos aumentados na axila à direita, sendo considerado categoria BI-RADS 5.

Nesse tempo, as lesôes na pele se acentuaram, tendo várias escoriaçoóes, descamação intensa e liquenificação, com lesôes ulceradas em nádegas (Figura 1). Notou intensa xerodermia e aumento da sensibilidade da pele em todo o corpo, ainda tendo forte prurido associado a calafrios frequentes. Foi encaminhada à FCecon em maio de 2018. Ao exame físico, apresentava tumor palpável em mama direita de aproximadamente 7 centímetros, com linfonodo axilar positivo móvel à direita. Após realização de exames complementares, o estadiamento clínico da paciente foi T3N1M0 e classificado em estágio IIIA. Os exames laboratoriais apresentaram anemia normocítica e normocrômica (hematócrito: $28,6 \%$ ), associada à eosinofilia (4\%), e proteína C-reativa aumentada (2,98 $\mathrm{mg} / \mathrm{dL}$ ), os demais exames laboratoriais estavam dentro dos padróes de normalidade, incluindo ureia, eletrólitos e IgE sérico.

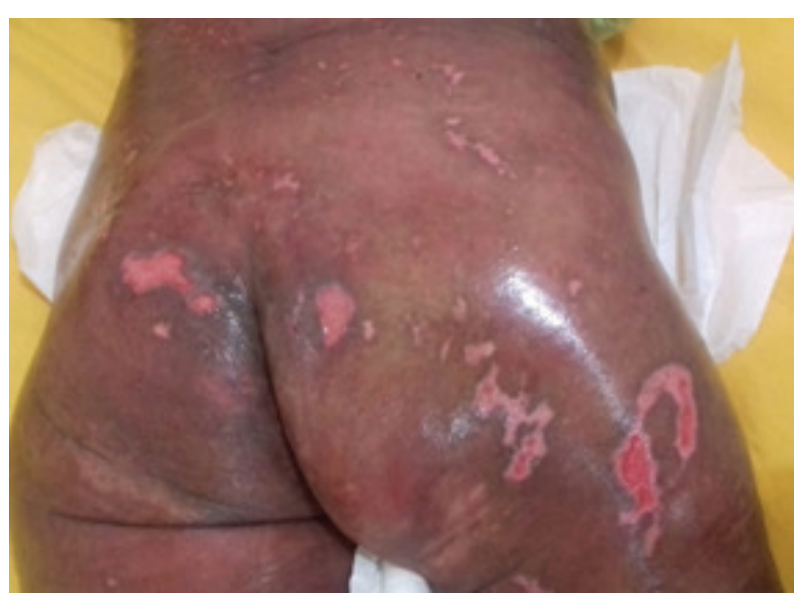

Figura 1. Lesões em nádegas

Ainda em maio de 2018, a biópsia da pele da lesão na regiāo da nádega direita revelou intenso infiltrado inflamatório mononuclear, superficial e perivascular, edema e ectasia vasculares presentes em derme papilar, 
além da presença de linfócitos $\mathrm{T}$ e $\mathrm{B}$, sem atipias. A biópsia da nádega esquerda (Figura 2) na mesma data também teve os mesmos achados. Na imuno-histoquímica, foi evidenciada a expressão de CD-31, CD-34, CD-20 e CD-3, o que favoreceu o diagnóstico de dermatite de caráter inflamatório, não havendo critérios para estabelecimento diagnóstico de linfoma cutâneo de células T ou psoríase.

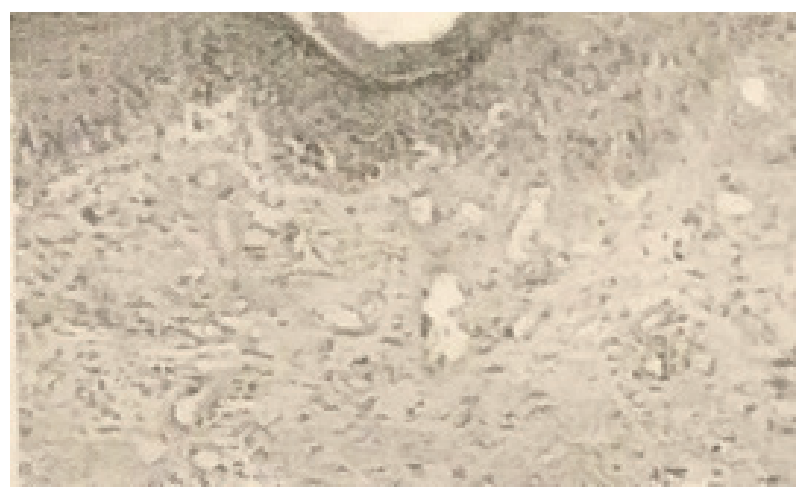

Figura 2. Biópsia de pele em região da nádega esquerda evidenciando lesão na derme, com acantose, paraceratose e discreta espongiose (HE x 10)

Durante os meses de maio e junho, referiu melhora parcial dos sintomas e diminuição de calafrios após início de hidroxizina.

Em agosto de 2018, a biópsia do nódulo da mama direita revelou que se tratava de um carcinoma invasor de mama e o exame de imuno-histoquímica mostrou que havia positividade para receptores de estrogênio $(10 \%)$ e para receptores de progesterona (40\%), elevado índice de proliferação com Ki-67 em 10\% das células, porém negativo para human epidermal growth factor receptor 2 (HER2), sendo caracterizado como subtipo molecular luminal A. Em setembro, iniciou a hormonioterapia com anastrozol.

Em novembro de 2018, realizou mastectomia com linfadenectomia axilar direita e em dezembro foi realizada uma reabordagem axilar por recidiva. Porém, não houve melhora da dermatite.

Após realização de tomografia de tórax em janeiro de 2019 com linfonodos axilares suspeitos, foi realizada biópsia de linfonodo axilar direito em fevereiro, sendo confirmada metástase. Em março deste mesmo ano, foi decidida uma nova reabordagem cirúrgica axilar, a qual foi realizada em abril. Em maio, iniciou tamoxifeno, dose diária de $20 \mathrm{mg}$, e no mês de junho foi interrompido, sendo iniciada a capecitabina (dose metrônica). Em agosto de 2019, referiu melhora do prurido e ressecamento de mucosas; apesar disso, a intensa xerodermia e a descamação generalizada persistiam.

Em consulta, em novembro de 2019, apresentava leve melhora do quadro com uso de loção hidratante e diminuiçáa do prurido, ainda sentia calafrios e referia dispneia ocasionalmente. Quanto ao exame físico, apresentava-se em regular estado geral, ativa, hipocorada e desidratada, sem hepatoesplenomegalia. $\mathrm{Na}$ pele apresentava apergaminhamento, além de lesóes em mucosa oral e edema de membros inferiores bilateral simétrico, sem cacifo. Apresentava hiperemia conjuntival, lesóes descamativas generalizadas, inclusive em couro cabeludo, além de lesões erosivas em membros inferiores, com surgimento de bolha em membro superior direito (Figura 3).

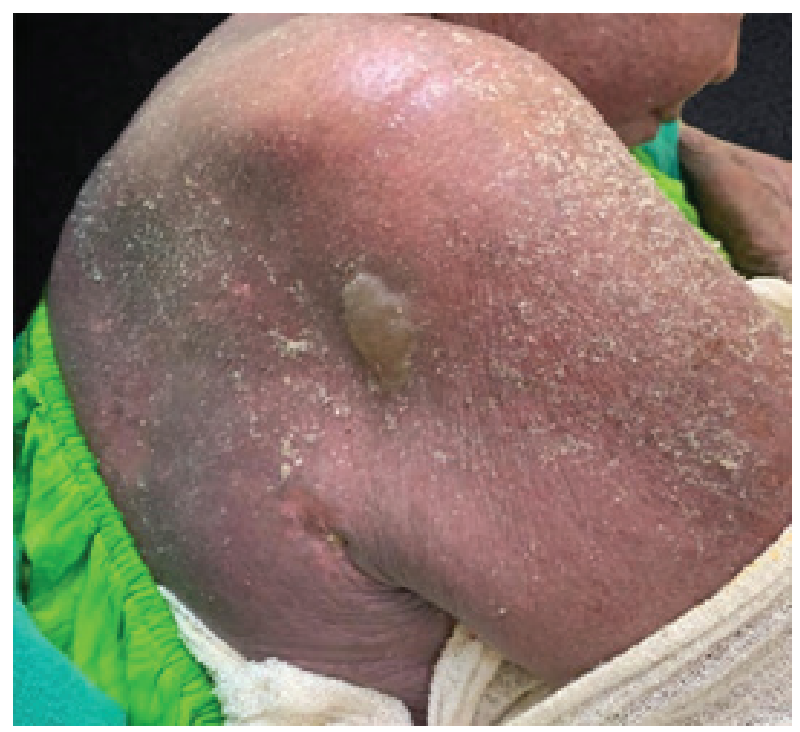

Figura 3. Bolha e descamação em região de membro superiores

Em fevereiro de 2020, após realização de tomografia de tórax, verificou-se derrame pleural maior à direita com linfonodomegalia e na tomografia de abdome foram visualizados nódulos hepáticos, sendo constatada a progressão da doença. Em abril, foi suspensa a capecitabina e iniciada a doxorrubicina lipossomal $49 \mathrm{mg}$. A paciente segue em acompanhamento ambulatorial.

\section{DISCUSSÃO}

Este caso é notável em virtude de ser um quadro atípico, visto que a dermatite esfoliativa é considerada uma doença rara, sendo de difícil tratamento e regressão das lesóes ${ }^{5,6}$. A eritrodermia é comumente associada a dermatoses preexistentes (psoríase, dermatite atópica ou dermatite de contato), reaçáo por fármacos, e ocasionalmente por tumores sólidos, porém $25 \%$ dos casos são idiopáticos ${ }^{7,8}$. Apesar de ser mais comumente associada a malignidades como leucemia e linfoma, também pode ocorrer em tumores laríngeos, gástricos, esofágicos, entre outros. As neoplasias devem ser descartadas precocemente, tendo a correlação clínica ${ }^{6,9}$. 
Os linfomas cutâneos de células $T$ são importantes diagnósticos diferenciais, sendo os principais tipos: a micose fungoide e a síndrome de Sézary (quando ocorre leucemização). A biópsia cutânea e citometria para células T malignas circulantes (células de Sézary) elucidam o diagnóstico, contudo, em fases precoces, podem não ser observadas por quantidade de células insuficientes ${ }^{10}$. Para a paciente em questão, os resultados da biópsia cutânea e da imuno-histoquímica não evidenciaram achados que estabelecessem o diagnóstico para essas doenças.

Em diversas manifestações cutâneas associadas a tumores malignos, incluindo eritrodermia, não há presença de células neoplásicas, mas possivelmente podem estar relacionadas à interaçáo entre citocinas (interleucinas $1,2,8)$ e a moléculas de adesão celular (VCAM-1, ICAM-1, E-selectina e P-selectina), levando à transmigração de linfócitos e células mononucleares ${ }^{11,12}$. Um infiltrado linfocítico (CD8 positivo e macrófagos), associado à disceratose do queratinócitos basais, é encontrado na junção dermoepidérmica ${ }^{12}$. Necrose epidérmica, vesiculações ou bolhas subepidérmicas podem ocorrer em situaçôes crônicas ${ }^{11,13}$.

Quanto ao quadro clínico, o elemento mais importante é a história clínica. A doença se apresenta de maneira insidiosa e gradual, exceto em casos de origem medicamentosa, começa com placas eritematosas que se espalham e podem afetar toda a pele ${ }^{8}$. Nas fases agudas, as descamaçôes são maiores e de aspecto crostoso, enquanto, na fase crônica, tendem a ser menores e mais secas; também pode haver hipo ou hiperpigmentação das regióes acometidas ${ }^{14}$, assim como na paciente deste estudo. Dos exames laboratoriais, é observado com frequência um aumento de IgE sérica, anemia, velocidade de hemossedimentação aumentada, eosinofilia, linfocitose e hipoproteinemia ${ }^{2}$. A paciente apresentava eosinofilia inicialmente e anemia normocítica e hipocrômica.

$\mathrm{Na}$ eritrodermia esfoliativa, o eritema aparece antes da descamação, que pode variar conforme a doença de base. Com a cronicidade do processo, pode ocorrer comprometimento de pelos (alopecia difusa em grau variável), unhas e surgimento de ectrópio. $\mathrm{O}$ paciente geralmente apresenta forte prurido, acarretando áreas de liquenificação e, eventualmente, erosôes com ocasional infecção. Por conta da perda de calor e eletrólitos, há crises de calafrios constantes, como a paciente do caso tinha ${ }^{13}$. Também, por conta do quadro, podem ocorrer complicaçóes bacterianas ${ }^{1}$. O quadro clínico da paciente deste estudo era compatível com eritrodermia e a biópsia da pele teve achados compatíveis com a doença (hiperceratose e acantose).

O tratamento primário da eritrodermia consiste em hidratação otimizada e correção dos distúrbios hidroeletrolíticos em regime de internação hospitalar ${ }^{7,8}$. Os corticosteroides tópicos com anti-histamínicos orais são adequados na maioria dos casos, porém, a reversão do quadro dependerá do agente causador, visto que as manifestaçôes geradas por medicamentos, após a interrupção, têm recuperação mais rápida ${ }^{12}$, enquanto, nas ocasionadas por malignidades, a remoçáo de tumores sólidos é imperativa ${ }^{7}$.

É importante ressaltar que, para pacientes com lesôes palpáveis de mama, a investigação diagnóstica deve ser realizada precocemente, visto que aumenta a chance de tratamento eficaz e reduz mortalidade, tendo a biópsia ambulatorial da mama um impacto significativo para a detecção precoce da doença $a^{15}$.

\section{CONCLUSÃO}

A importância deste caso consiste em trazer novas evidências acerca do tema e ressaltar que a eritrodermia também pode estar associada à neoplasia maligna da mama, sendo um importante diagnóstico etiológico a ser considerado, principalmente naqueles pacientes em que não há dermatoses preexistentes.

\section{CONTRIBUIÇÕES}

Maykom de Lira Barbosa, Yara Ayami Mattos Abe e Hilka Flávia Barra do Espírito Santo Alves Pereira contribuíram substancialmente na concepção e planejamento do estudo; na análise e interpretação dos dados; e na redação e revisão crítica. Fábio de Queiroz Medeiros contribuiu substancialmente na análise e interpretação dos dados; e na redação. Todos os autores aprovaram a versáo final a ser publicada.

\section{DECLARAÇÃO DE CONFLITO DE INTERESSES}

Nada a declarar.

\section{FONTES DE FINANCIAMENTO}

Não há.

\section{REFERÊNCIAS}

1. Okoduwa C, Lambert W, Schwartz RA, et al. Erythroderma: review of a potentially life -threatening dermatosis. Indian J Dermatol [Internet]. 2009 [cited 2020 June 18];54(1):1-6. Available from: http://www.eijd.org/text.asp?2009/54/1/1/48976

2. Fernandes NC, Pereira FSM, Maceira JP, et al. Eritrodermia: estudo clínico-laboratorial e histopatológico de 170 casos. An Bras Dermatol. 2008;83(6):526-32. doi: https://doi.org/10.1590/S0365-05962008000600005 
3. Tan GFL, Kong YL, Tan ASL, et al. Causes and features of erythroderma. Ann Acad Med Singap [Internet]. 2014 [cited 2020 June 23];43(8):391-4. Available from: https://pubmed.ncbi.nlm.nih.gov/25244987. Free full text article. PMCID: 25244987.

4. Akhyani M, Ghodsi ZS, Toosi S, et al. Erythroderma: a clinical study of 97 cases. BMC Dermatol. 2005;5:5. doi: https://doi.org/10.1186/1471-5945-5-5

5. Megna M, Sidikov AA, Zaslavsky DV, et al. The role of histological presentation in erythroderma. Int J Dermatol. 2017;56(4):400-4. doi: https://doi. org/10.1111/ijd.13488

6. Altiner A, Chu J, Patel R, et al. Erythroderma of unknown etiology. Dermatol Online J [Internet]. 2011 [cited 2020 June 20];17(10):6. Available from: http:// www.ncbi.nlm.nih.gov/pubmed/22031632. Free full text article. PMCID: 22031632.

7. Protopsaltis I, Drossou A, Katsantonis I, et al. Breast cancer presenting as paraneoplastic erythroderma: an extremely rare case. Case Rep Med. 2014;2014:351065. doi: https://doi.org/10.1155/2014/351065

8. Cuellar-Barboza A, Ocampo-Candiani J, HerzRuelas ME. A practical approach to the diagnosis and treatment of adult erythroderma. Actas Dermosifiliogr. 2018;109(9):777-90. doi: https://doi.org/10.1016/j. adengl.2018.05.033

9. Silva JA, Mesquita KC, Igreja ACSM, et al. Paraneoplastic cutaneous manifestations: concepts and updates. An Bras Dermatol. 2013;88(1):09-22. doi: http://doi. org/10.1590/S0365-05962013000100001

10. Olsen E, Vonderheid E, Pimpinelli N. Revisions to the staging and classification of mycosis fungoides and Sezary syndrome: a proposal of the International Society for Cutaneous Lymphomas (ISCL) and the cutaneous lymphoma task force of the European Organization of Research and Treatment of Cancer (EORTC). Blood. 2007;110(6):1713-22. doi: http://doi.org/10.1182/ blood-2007-03-055749

11. Sigurdsson V, Vries IJ, Toonstra J, et al. Expression of VCAM-1, ICAM-1, E-selectin, and P-selectin on endothelium in situ in patients with erythroderma, mycosis fungoides and atopic dermatitis. J Cutan Pathol. 2000;27(9):436-40. doi: https://doi.org/10.1034/ j.1600-0560.2000.027009436.x

12. Schwartz RA, McDonough PH, Lee BW. Toxic epidermal necrolysis: Part II. Prognosis, sequelae, diagnosis, differential diagnosis, prevention, and treatment. J Am Dermatol. 2013;69(2):187.e1-16. doi: http://doi. org/10.1016/j.jaad.2013.05.002

13. Yacoub MR, Berti A, Campochiaro C, et al. Drug induced exfoliative dermatitis: State of the art. Clin Mol Allergy. 2016;14(1):9. doi: https://doi.org/10.1186/ s12948-016-0045-0
14. César A, Cruz M, Mota A, et al. Erythroderma. A clinical and etiological study of 103 patients. J Dermatol Case Rep. 2016;10(1):1-9. doi: http://doi.org/10.3315/ jdcr.2016.1222

15. Tomazelli JG, Migowski A, Ribeiro CM, et al. Avaliação das ações de detecção precoce do câncer de mama no Brasil por meio de indicadores de processo: estudo descritivo com dados do Sismama, 2010-2011. Epidemiol Serv Saúde. 2017;26(1):61-70. doi: http:// doi.org/10.5123/s1679-49742017000100007

Recebido em 17/7/2020 Aprovado em 3/9/2020 